\title{
Comparação Seriada da Miniavaliação Nutricional em Idosos Institucionalizados
}

\author{
Serial Comparison of the Nutritional Small evaluation in \\ Institutionalized Elderly
}

\author{
Jamile Ceolin* \\ Loiva Beatriz Dallepiane**
}

\section{Resumo}

Os idosos representam o segmento da população que mais cresce no mundo, e, no Brasil, o envelhecimento populacional está ocorrendo de forma rápida e progressiva. Apesar de ser um processo natural, o envelhecimento submete o organismo a diversas alterações, podendo repercutir diretamente no estado nutricional do idoso. Com base no exposto, este estudo tem como objetivo comparar o estado nutricional utilizando a Miniavaliação nutricional de idosos moradores em uma instituição de longa permanência, em uma cidade no interior do RS, no período de 2013 a 2014. Trata-se de um estudo quantitativo com análise de dados retrospectivos, extraídos dos prontuários de idosos institucionalizados de 2013 e 2014, utilizando a Miniavaliação Nutricional (MAN) como instrumento da pesquisa. A população de estudo foi constituída por 36 idosos, totalizando um percentual de $66,7 \%$ do sexo feminino. O diagnóstico obtido por meio da Miniavaliação nutricional observou que a maioria dos idosos institucionalizados apresentou risco nutricional em 2013 (61,1\%), bem como em 2014 (58,3\%). Já em relação à desnutrição o percentual aumentou de 16,7\% para 25\% no ano de 2013 para 2014. Em suma, por ser um grupo nutricionalmente vulnerável, principalmente por se tratar de idosos institucionalizados, as avaliações seriadas se revestem de importância no sentido de propor intervenção para os casos com alterações nutricionais.

Palavras-chave: Miniavaliação nutricional. Idoso. Saúde do idoso institucionalizado.

\begin{abstract}
Old people represent the part of the population that increases more in the world, and, in Brazil, the population's aging happens in a progressive and fast way. In spite of being a natural process, aging submits the human body to many changes and it can influence directly on the elderly's nutritional status. Based on the above, this study aims to compare the nutritional status using the elderly's nutritional minivaluation in a long stay institution, in a town from Rio Grande do Sul, between 2013 and 2014. This is a quantitative study with retrospective data analysis, analyzing information from 2013 and 2014, extracted from the institutionalized elderly's records using Miniavaliação Nutrition (MNA) as a research instrument. The population of the research was formed by 36 old people, totaling a percentile of $66,7 \%$ of women. The diagnosis got from the nutritional minivaluation observed that most institutionalized elderly showed nutritional risk in $2013(61,1 \%)$ as well as in 2014 (58,3\%). In relation to innutrition, in 2013 the percentage was 16,7\% and in 2014 it increased to $25 \%$. In short,for being a group vulnerable nutricionalty, mainly for taking care of institutionalized seniors, the seriate evaluations are covered of importance in the sense of proposing intervention for the cases with alterations nutricionals.
\end{abstract}

Keywords: Mini Nutritional Assessment. Elderly. Health of institutionalized elderly.

DOI: $10.15343 / 0104-7809.20164003327333$

\footnotetext{
*Universidade Federal de Santa Maria-UFSM. Santa Maria - RS, Brasil. Email: jamileceolin@yahoo.com.br

** Universidade Federal de Santa Maria-UFSM. Santa Maria - RS, Brasil. Email: loiva.dallepiane@hotmail.com

Os autores declaram não haver conflitos de interesse
} 


\section{INTRODUÇÃO}

Os idosos representam o segmento da população que mais cresce no mundo, e, no Brasil, o envelhecimento populacional está ocorrendo de forma rápida e progressiva. Segundo o Instituto Brasileiro de Geografia e Estatística, a população de idosos representa atualmente um contingente de quase 15 milhões de pessoas com 60 anos ou mais de idade $(8,6 \%$ da população brasileira). Dados do Censo Demográfico de 2010 revelam um aumento da população com 65 anos ou mais, que era de $4,8 \%$ em 1991, passando a 5,9\% em 2000 e chegando a 7,4\% em 2010. No Rio Grande do Sul, segundo este censo, o percentual da população acima de 60 anos já atingiu $13,65 \%{ }^{1}$.

O envelhecimento populacional é considerado um fenômeno local, regional, nacional e global, uma vez que esse processo iniciou-se nos países desenvolvidos e espalhouse para os países em desenvolvimento, em resposta à diminuição das taxas de fecundidade, natalidade e mortalidade e pelo aumento da esperança média de vida ao nascer ${ }^{2,3}$.

O envelhecimento, apesar de ser um processo natural, submete o organismo a diversas alterações, o qual é marcado pela diminuição progressiva da atividade celular, perda da capacidade funcional e modificações metabólicas. Essas modificações consideráveis no organismo do indivíduo levam a perdas sensoriais, ausência de dentes e/ou próteses mal adaptadas, diminuição da secreção gástrica, motilidade gastrointestinal e redução da taxa metabólica basal, que pode repercutir diretamente no estado nutricional do idoso ${ }^{4}$.

Com a presença de alguns agravantes genéticos e/ou ambientais, alguns idosos têm um declínio do estado de saúde, tornando-se mais frágeis e dependentes de cuidados externos, e, com isso, há a procura por instituições de longa permanência para idosos (ILPI) ${ }^{3}$.

Sperotto e Spinelli ${ }^{5}$ destacam a importância da realização da avaliação do estado nutricional em idosos, visto que é essencial para se detectar precocemente a desnutrição além de facilitar o trabalho de profissionais no tratamento para recuperar alterações nutricionais e contribuir com a promoção da saúde dos idosos e consequentemente com o restabelecimento de suas funções orgânicas, melhorando a qualidade de vida.

A Miniavaliação nutricional foi desenvolvida em 1989, com o objetivo de avaliar o risco nutricional em idosos, assim como para identificar aqueles que pudessem se beneficiar de intervenção dietoterápica precoce.

A MAN, em sua versão original é composta de 18 tópicos, que englobam avaliação antropométrica, dietética e clínica e até mesmo a autopercepção da saúde e estado nutricional, podendo ser utilizada tanto para triagem como para apreciação diagnóstica. Esse instrumento leva em consideração o estado nutricional, o estado de saúde, a fragilidade e eventuais doenças que acometem os idosos ${ }^{7}$.

Portanto, considerando a importância da nutrição como um fator primordial na qualidade de vida, bem como diagnóstico precoce de distúrbios nutricionais nessa população, este estudo objetivou verificar a alteração do estado nutricional pela Miniavaliação nutricional (MNA) de idosos moradores em uma instituição de longa permanência no período de um ano.

\section{METODOLOGIA}

Trata-se de um estudo quantitativo com análise de dados retrospectivos extraídos dos prontuários de uma instituição de longa permanência para idosos (ILPI), no período de 2013 e 2014. A população foi constituída de 36 idosos institucionalizados com idade acima de 60 anos e que realizaram a avaliação nutricional nos dois anos consecutivos, sendo excluídos 6 idosos dentre eles os que não realizaram a avaliação nutricional em 2013 ou 2014, os acamados e os que foram a óbito no período do estudo.

Na época da coleta dos dados, a equipe da ILPI era composta pela coordenadora da instituição, enfermeiros, técnicos em enfermagem e auxiliar de serviços gerais, sendo que as avaliações nutricionais eram realizadas por acadêmicos bolsistas do curso de nutrição. Atualmente, essa ILPI conta com um profissional nutricionista no acompanhamento do estado nutricional. 
Para a avaliação do estado nutricional dos idosos, foi realizado a avaliação antropométrica: peso, estatura para o cálculo do IMC e também avaliado pela Miniavaliação nutricional (MAN). A MANé uma ferramenta de avaliação geriátrica rápida e composta por um questionário com 18 questões objetivas, que avaliam as medidas antropométricas, contemplando a avaliação global, um questionário dietético simples e a avaliação subjetiva. A MAN é dividida em duas etapas: uma avaliação de triagem, com seis questões que englobam alterações da ingestão alimentar (por perda de apetite, problemas digestivos ou difficuldade de mastigação ou deglutição), perda de peso, mobilidade, ocorrência de estresse psicológico ou doença aguda, problemas neuropsicológicos e Índice de Massa Corporal (IMC).

A segunda etapa da MAN consiste na avaliação global, contendo 12 questões relativas ao modo de vida, lesões de pele ou escaras, medicação, avaliação dietética (perguntas relativas ao número de refeições, ingestão de alimentos e líquidos e autonomia na alimentação), autoavaliação (autopercepção da saúde e da condição nutricional), e a antropometria é então complementada com o perímetro do braço e da panturrilha.

Cada questão contém um valor numérico que contribui para o resultado final do escore. A primeira etapa da MAN possui um subtotal do escore de 14 pontos. Caso o idoso obtiver um escore maior ou igual a 12 pontos, indica que não apresenta risco nutricional, não sendo necessário dar continuidade ao questionário; entretanto, para fins de acompanhamento dos idosos institucionalizados, na ILPI é realizado todooquestionáriodaMANindependentemente do resultado da triagem, permitindo, assim, a comparação das informações com os anos subsequentes, visando identificar em qual dos itens pode haver alguma alteração.

Quando o escore for menor ou igual a 11, continua-se com a segunda etapa da avaliação, para determinar o escore do indicador de desnutrição. Essa etapa consiste na avaliação global e possui escore máximo de 16 pontos. A soma total dos escores chega a um valor máximo de 30 pontos com a seguinte interpretação dos resultados: desnutrição (< 17 pontos) e risco de desnutrição (17 a 23,5 pontos).
Araújo et $\mathrm{al}^{18}$, ao analisar a validação do método de triagem nutricional da MAN, obteve sensibilidade de 96\%, especificidade de 98\% e o valor prognóstico para desnutrição de 97\%. O método de avaliação, além de apresentar alta sensibilidade, especi-aficidade e valor prognóstico citado, trata-se de um instrumento de triagem validada para idosos. Para as questões subjetivas, excetuando-se as variáveis antropométricas, a MAN atribui pontos que variam de zero a três, dependendo da questão em particular, que indica que, quanto mais perto de zero, pior é a situação do idoso e, quanto mais alta a pontuação, melhor é a condição.

Para os indicadores antropométricos de Índice de Massa Corporal (IMC), Circunferência do Braço (CB) e Circunferência da Panturrilha (CP) utilizados na MAN, o próprio instrumento atribui uma pontuação. Os dados foram processados em uma planilha do Excel e analisados no software SPSS 18.0. Foi utilizada a estatística descritiva, "test t-Student" e teste "qui-quadrado" e considerou-se o valor de p menor que 0,05 como estatisticamente significativo.

A presente pesquisa foi conduzida de acordo com os preceitos éticos, sendo aprovada pelo Comitê de Ética em Pesquisa (CEP) da Universidade Federal de Santa Maria (USFM), sob o número do CAAE 29862314.0.0000.5346, sendo seguidos todos os preceitos éticos da Resolução 466/12 do Conselho Nacional de Saúde, do Ministério da Saúde (CNS/MS).

\section{RESULTADOS}

A população de estudo foi constituída por 36 idosos residentes em uma ILPI de uma cidade do interior do RS. A amostra totalizou um percentual de $66,7 \%$ do sexo feminino $(n=24)$. O tempo de permanência dos idosos na instituição variou de menos de um a 29

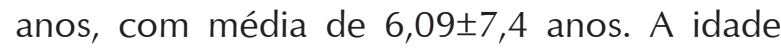
variou de 60 a 98 anos, com média 78,47士10,6 anos. Entre os idosos, $47,2 \%$ situavam-se em 80 anos ou mais, $27,8 \%$ com idade entre 70 e 79,9 anos e $25 \%$ de 60 a 69,9 anos. Os dados foram retirados dos prontuários dos idosos institucionalizados (Tabela 1). 
Tabela 1 - Características da população de idosos residentes em uma ILPI. Palmeira das Missões/RS, 2014.

\begin{tabular}{|c|c|c|c|c|}
\hline & & \multicolumn{2}{|c|}{ Sexo } & \multirow[b]{2}{*}{$\mathbf{p}^{*}$} \\
\hline & & $\begin{array}{c}\text { Feminino } \\
\quad \mathbf{N}(\%)\end{array}$ & $\begin{array}{c}\text { Masculino } \\
\text { N (\%) }\end{array}$ & \\
\hline \multirow[t]{4}{*}{ Tempo ILPI } & Menos de 1 ano & $4(80,0)$ & $1(20,0)$ & \\
\hline & 1 a 4,99 anos & $9(50,0)$ & $9(50,0)$ & 0,14 \\
\hline & 5 anos a 9,99 anos & $5(100,0)$ & $0(0,0)$ & \\
\hline & $\geq 10$ anos & $6(75,0)$ & $2(25,0)$ & \\
\hline \multirow[t]{3}{*}{ Idade } & $60-69,9$ anos & $8(88,9)$ & $1(11,1)$ & \\
\hline & 70 - 79,9 anos & $6(60,0)$ & $4(40,0)$ & 0,26 \\
\hline & $\geq 80$ anos & $10(58,8)$ & $7(41,2)$ & \\
\hline
\end{tabular}

${ }^{*} \mathrm{p}<0,05=$ estatisticamente significativo

Na tabela 2, estão descritos os dados referentes ao gênero, à idade e à média dos resultados em comparação dos anos de 2013 e 2014 quanto às variáveis antropométricas. A média da estatura foi menor quanto maior a idade, especialmente no sexo feminino. A média do peso teve um comportamento similar, ou seja, a média foi menor nos idosos com faixa etária acima de 80 anos em ambos os sexos e nos dois períodos consecutivos, no entanto, os idosos do sexo masculino acima de 80 anos apresentaram um aumento da média do peso em 2014, comparado com 2013. Em relação ao IMC no sexo feminino, a média diminuiu no período de um ano em todas as faixas etárias, não obstante, no sexo masculino houve um aumento da média em 2014 nos idosos de faixa etária acima de 70 anos. Já a CB, no sexo feminino, observou-se uma diminuição gradual conforme aumentou a idade e tendo aquelas acima de 80 anos o valor mais baixo. Nos homens, com idade acima de 80 anos registrou um declínio da média em 2014, comparado com 2013. A comparação da média da CP de ambos os sexos não apresentou significância estatística.

Tabela 2 - Evolução dos indica_dores antropométricos por faixa etária e sexo dos idosos institucionalizados durante um ano. Palmeira das Missões/RS, 2014.

\begin{tabular}{|c|c|c|c|c|c|c|c|}
\hline \multirow{3}{*}{ Variáveis } & \multirow{3}{*}{ Sexo } & \multicolumn{6}{|c|}{ Idade (anos) } \\
\hline & & \multicolumn{2}{|c|}{60 a $69,9(n=9)$} & \multicolumn{2}{|c|}{70 a $79,9(n=10)$} & \multicolumn{2}{|c|}{70 a $79,9(n=10)$} \\
\hline & & $\begin{array}{c}2013 \\
\text { Média } \pm \text { DP }\end{array}$ & $\begin{array}{c}2014 \\
\text { Média } \pm D P\end{array}$ & $\begin{array}{c}2013 \\
\text { Média } \pm \mathrm{DP}\end{array}$ & $\begin{array}{c}2014 \\
\text { Média } \pm D P\end{array}$ & $\begin{array}{c}2013 \\
\text { Média } \pm D P\end{array}$ & $\begin{array}{c}2014 \\
\text { Média } \pm \text { DP }\end{array}$ \\
\hline $\begin{array}{l}\text { Estatura } \\
(\mathbf{c m})\end{array}$ & $\begin{array}{l}\mathrm{F} \\
\mathrm{M}\end{array}$ & $\begin{array}{l}155 \\
160\end{array}$ & $\begin{array}{l} \pm 7,4 \\
\pm 0,0\end{array}$ & $\begin{array}{c}151 \\
16\end{array}$ & $\begin{array}{c} \pm 10,2 \\
\pm 3,3\end{array}$ & $\begin{array}{r}146 \\
155,\end{array}$ & $\begin{array}{l} \pm 9,2 \\
\pm 13,4\end{array}$ \\
\hline Peso (kg) & $\begin{array}{l}F \\
M\end{array}$ & $\begin{array}{l}53,8 \pm 10,4 \\
66,8 \pm 0,0\end{array}$ & $\begin{array}{l}52,6 \pm 11,2 \\
65,0 \pm 0,0\end{array}$ & $\begin{array}{l}55,2 \pm 7,6 \\
60,7 \pm 13,3\end{array}$ & $\begin{array}{l}53,9 \pm 7,7 \\
65,0 \pm 15,8\end{array}$ & $\begin{array}{l}47,3 \pm 16,4 \\
50,5 \pm 10,4\end{array}$ & $\begin{array}{l}45,2 \pm 19,1 \\
54,4 \pm 14,6\end{array}$ \\
\hline $\begin{array}{l}\text { IMC } \\
\left(\mathrm{kg} / \mathrm{m}^{2}\right)\end{array}$ & $\begin{array}{l}\mathrm{F} \\
\mathrm{M}\end{array}$ & $\begin{array}{l}22,6 \pm 5,5 \\
26,1 \pm 0,0\end{array}$ & $\begin{array}{l}22,0 \pm 5,3 \\
25,4 \pm 0,0\end{array}$ & $\begin{array}{l}24,3 \pm 3,5 \\
23,7 \pm 4,4\end{array}$ & $\begin{array}{l}23,8 \pm 3,9 \\
25,4 \pm 5,6\end{array}$ & $\begin{array}{l}22,0 \pm 6,6 \\
20,9 \pm 3,1\end{array}$ & $\begin{array}{l}21,2 \pm 8,2 \\
22,6 \pm 5,7\end{array}$ \\
\hline CB $(\mathrm{cm})$ & $\begin{array}{l}\mathrm{F} \\
\mathrm{M}\end{array}$ & $\begin{array}{l}28,4 \pm 3,6 \\
28,0 \pm 0,0\end{array}$ & $\begin{array}{l}27,1 \pm 3,8 \\
25,0 \pm 0,0\end{array}$ & $\begin{array}{l}26,8 \pm 1,6 \\
26,1 \pm 3,8\end{array}$ & $\begin{array}{l}26,3 \pm 2,1 \\
29,0 \pm 6,4\end{array}$ & $\begin{array}{l}24,8 \pm 5,1 \\
24,1 \pm 2,5\end{array}$ & $\begin{array}{l}23,8 \pm 5,2 \\
22,8 \pm 2,2\end{array}$ \\
\hline $\mathrm{CP}(\mathrm{cm})$ & $\begin{array}{l}\mathrm{F} \\
\mathrm{M}\end{array}$ & $\begin{array}{l}30,8 \pm 3,2 \\
32,0 \pm 0,0\end{array}$ & $\begin{array}{l}31,9 \pm 3,5 \\
32,0 \pm 0,0\end{array}$ & $\begin{array}{l}31,3 \pm 3,3 \\
32,1 \pm 3,0\end{array}$ & $\begin{array}{l}31,5 \pm 3,3 \\
33,2 \pm 3,0\end{array}$ & $\begin{array}{l}28,0 \pm 4,6 \\
29,9 \pm 4,3\end{array}$ & $\begin{array}{l}27,6 \pm 5,1 \\
30,4 \pm 4,3\end{array}$ \\
\hline
\end{tabular}

$\mathrm{IMC}=$ índice de massa corporal; $\mathrm{CB}=$ circunferência do braço; $\mathrm{CP}=$ circunferência da panturrilha 
Analisando o escore de triagem em 2013, $77,8 \%(n=28)$ dos idosos apresentaram escore $\leq 11$; já em 2014, a porcentagem diminuiu para $72,2 \%(n=26)$, indicando que a grande maioria dos idosos desta ILPI continuaram em risco nutricional.

A figura 1 demonstra o escore de indicação de desnutrição segundo a MAN dos idosos em 2013 e 2014, observando-se que, tanto em 2013 como em 2014, a maioria (61,1\% e $58,3 \%$ respectivamente) dos idosos institucionalizados apresentou risco nutricional (17-23,5 pontos).

A tabela 3 apresenta a média das variáveis do estudo de 2013 e 2014, o intervalo de confiança e a diferença da média. Os resultados não apresentaram diferenças estatisticamente significativas entre os anos.

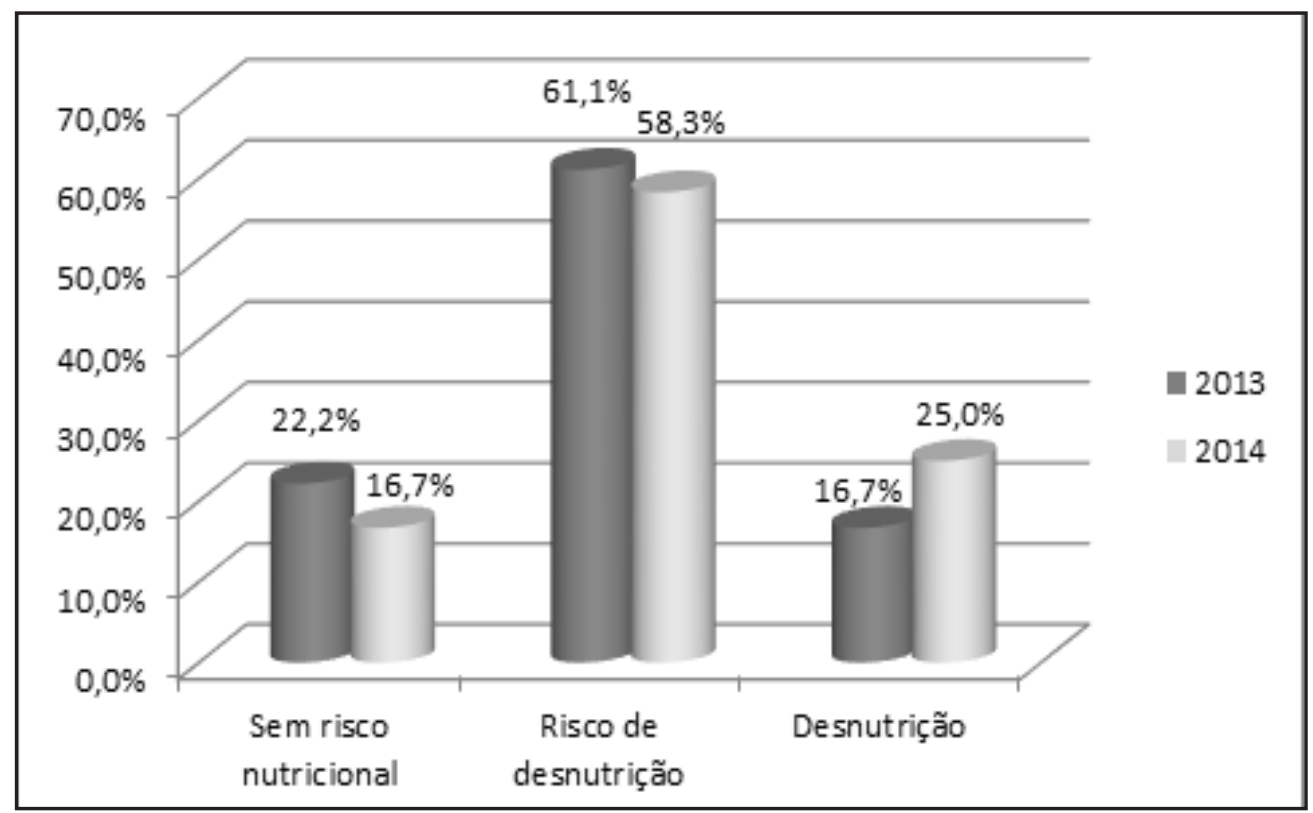

Figura 1 - Avaliação do Estado Nutricional dos idosos institucionalizados de acordo com os escores da Miniavaliação nutricional (MAN) entre 2013 e 2014. Palmeira das Missões/RS, 2014.

Tabela 3 - Comparação dos resultados da antropometria e do escore da MAN dos anos de 2013 e 2014 em idosos de uma ILPI (RS, 2014)

\begin{tabular}{lcccc}
\hline \multicolumn{1}{c}{ Variáveis } & Média $\mathbf{2 0 1 3}$ & Média $\mathbf{2 0 1 4}$ & $\begin{array}{c}\text { Diferença } \\
\text { da Média }(\mathbf{I C})\end{array}$ & P \\
\hline Peso $(\mathbf{k g})$ & $52,69 \pm 12,5$ & $52,85 \pm 14,9$ & $0,16 \pm 5,9(-1,84$ a 2,16$)$ & 0,87 \\
$\mathbf{I M C}\left(\mathbf{k g} / \mathbf{m}^{2}\right)$ & $22,59 \pm 4,9$ & $22,64 \pm 5,9$ & $0,05 \pm 2,59(-0,82$ a 0,93$)$ & 0,90 \\
$\mathbf{C P}(\mathbf{c m})$ & $30,08 \pm 3,9$ & $30,48 \pm 4,3$ & $0,39 \pm 1,22(-0,01$ a 0,81$)$ & 0,06 \\
$\mathbf{C B}(\mathbf{c m})$ & $26,01 \pm 3,8$ & $25,36 \pm 4,4$ & $-0,65 \pm 2,6(-1,54$ a 0,24$)$ & 0,14 \\
Escore da & $10,65 \pm 1,8$ & $10,26 \pm 2,4$ & $-0,38 \pm 2,19(-1,13$ a 0,35$)$ & 0,29 \\
avaliação global & $9,66 \pm 2,4$ & $9,22 \pm 3,1$ & $-0,44 \pm 3,03(-1,47$ a 0,58$)$ & 0,38 \\
Escore da triagem & $20,31 \pm 3,8$ & $19,58 \pm 5,1$ & $-0,73 \pm 4,14(-2,13$ a 0,66$)$ & 0,29 \\
Escore total & &
\end{tabular}




\section{DISCUSSÃo}

A apresentação dos dados em relação ao perfil demográfico dos idosos institucionalizados deste estudo destaca uma maior participação do sexo feminino e acima de 80 anos. A institucionalização parece ser, em sua maioria, uma questão feminina, sendo também verificada em outros estudos ${ }^{9}$. Segundo o Instituto Brasileiro de Geografia e Estatística IBGE, ${ }^{10}$ no Brasil, em média, as mulheres vivem oito anos a mais que os homens. Affeldt ${ }^{2}$ afirma em seu estudo que o crescimento da população idosa é cada vez mais evidente, no qual o sexo feminino está se sobressaindo sobre o sexo masculino, evidenciando no envelhecimento a ocorrência da "feminização da velhice".

Em relação ao estado nutricional diagnosticado pela MAN, verificou-se que a desnutrição nos idosos institucionalizados aumentou de 2013 para 2014. Outro estudo realizado com idosos independentes de uma ILPI utilizando o mesmo instrumento de avaliação, observou que 35\% dos idosos estavam em desnutrição, $65 \%$ da amostra estavam em risco nutricional e nenhum idoso, dentre os avaliados, em bom estado nutricional, esses resultados podem ser explicados pelo fato de serem excluídos do estudo os idosos com problemas de surdez, mudez, deficiência mental e dependentes ${ }^{5}$.

Dados de outra pesquisa realizada em um asilo da cidade de Rio Grande- RS, divergiu destes resultados, sendo que foi encontrado mais da metade dos idosos institucionalizados bem-nutridos, enquanto que apenas 10,7\% estavam em desnutrição, fato este que pode estar relacionado às condições da ILPI e de uma equipe multiprofissional presente e atuante. ${ }^{12}$

Analisando o escore de triagem, Gomes, Pinto e Soar ${ }^{13}$, em seu estudo encontrou que $58,8 \%$ $(n=20)$ dos idosos apresentaram um escore $\leq 11$ pontos, sendo este inferior ao encontrado no presente trabalho, sugerindo um maior risco nutricional dos idosos do presente estudo. No levantamento efetuado por Silva et $\mathrm{al}^{14}$ relatou que, dos idosos institucionalizados avaliados pela MAN, 34\% atingiram o escore igual ou superior a 12, descartando a possibilidade de desnutrição.

Quanto ao IMC, Volpini e Frangella ${ }^{15}$, em seu estudo com idosos em ILPI, encontraram a média do IMC de $24,6 \pm 2,2 \mathrm{~kg} / \mathrm{m}^{2}$ no sexo masculino e $23,9 \pm 3,5 \mathrm{~kg} / \mathrm{m}^{2}$ no feminino, o que caracterizou uma amostra eutrófica; entretanto, no grupo masculino acima de 95 anos e no feminino após os 100 anos, os valores encontraram-se abaixo do valor de referência. Os valores da média do IMC em ambos os sexos são superiores ao do presente estudo, ou seja a população estudada se caracteriza por ser mais emagrecida comparada com os idosos do estudo referido.

Quanto à CB, um estudo que avaliou o estado nutricional dos idosos registrou a presença de desnutrição em 54,1\% dos idosos institucionalizados ${ }^{16}$. A diminuição da circunferência do braço reflete na redução de massa muscular e tecido subcutâneo, um método simples para avaliar a desnutrição no idoso, visto que o braço se compõe essencialmente por gordura e músculo ${ }^{17}$.

Sperotto e Spinelli ${ }^{5}$ afirmam que os idosos brasileiros acima de 60 anos de idade têm um risco maior de apresentar desnutrição, um percentual em torno de 52,8\%. Na região Sul, a prevalência de desnutrição é de 38,9\%, enquanto o risco de morte por desnutrição no Brasil, durante a velhice, comparado a outros países, é de $71 \%$, maior do que nos EUA e $32,1 \%$ maior do que na Costa Rica.

\section{CONCLUSÃO}

O acompanhamento seriado do estado nutricional de idosos institucionalizados é uma área pouco explorada; dessa forma, a escassez da literatura com informações comparativas da avaliação nutricional em diferentes períodos acarretou dificuldade em encontrar pesquisas com as quais os dados deste estudo pudessem ser comparados.

As principais conclusões deste estudo concentram-se na feminização do envelhecimento na ILPI e numa grande parcela de idosos acima de 80 anos.

No resultado da MAN, denominado como escore de indicação de desnutrição dos idosos avaliados entre 2013 e 2014, foi possível observar que o índice de risco nutricional prevaleceu entre os idosos institucionalizados, 
com indicação do aumento da desnutrição.

Por ser considerado um grupo nutricionalmente vulnerável, principalmente idosos que residem em ILPI, seria necessária uma adequação nutricional e uso de práticas educativas, com intuito de melhorar o perfil nutricional e consequentemente a qualidade de vida dos idosos.
Além disso, é de extrema necessidade a realização de acompanhamentos periódicos do estado nutricional, para que se possa detectar precocemente riscos nutricionais e fazer uma intervenção adequada. Neste sentido, a MAN cumpre seu objetivo pela facilidade e boa precisão de identificar idosos em risco nutricional.

\section{REFERÊNCIAS}

1. Brasil. Fundação Instituto Brasileiro de Geografia e Estatística. Censo Demográfico 2010. [Acesso em 2014 mai 18]. Disponível em: URL: < http://www.ibge.gov.br/home/estatistica/populacao/censo2010/>. Acesso em: 18mai.2014

2. Affeldt MAF.O asilo enquanto espaço e lugar: a institucionalização da velhice em Santa Maria-RS. [tese]. Santa Maria: Universidade Federal de Santa Maria; 2013.

3. Neves HMF. Causas e Consequências da Institucionalização de Idosos. Estudo tipo série de casos. [tese]. Covilhã: Universidade da Beira Interior; 2012.

4. Lima RSS; Lima RS; ALMEIDA ASSS. Projeto saúde: perfil alimentar e nutricional de idosas de um município do interior do Ceará. Revista Brasileira de Nutrição Esportiva2013 jan-fev;7(37):4-12.

5. Sperotto FM, Spinelli RB.Avaliação nutricional em idosos independentes de uma Instituição de Longa Permanência no Município de Erechim-RS, Perspectiva 2010;34(125):105-16.

6. Brasil. Ministério do Desenvolvimento Social e Combate à Fome. Política Nacional do Idoso. Lei no 8.842, de janeiro de 1994. Brasília, 2010.

7. Sousa VMC, Guariento ME. Avaliação do idoso desnutrido. Rev Bras Clin Med 2009;7(1):46-9.

8. Brasil. Ministério da Saúde. Secretaria de Atenção à Saúde. Departamento de Atenção Básica. Orientações para a coleta e análise de dados antropométricos em serviços de saúde: Norma Técnica do Sistema de Vigilância Alimentar e Nutricional - SISVAN / Ministério da Saúde, Secretaria de Atenção à Saúde, Departamento de Atenção Básica. - Brasília: Ministério da Saúde, 2011.76 p.: il. - (Série G. Estatística e Informação em Saúde)

9. Menezes TN, Marucci MFN.Avaliação antropométrica de idosos residentes em Instituições de Longa Permanência de Fortaleza-CE. Revista Brasileira Geriatria Gerontologia 2010 jan-ago;13(2):235-43.

10. Brasil. Fundação Instituto Brasileiro de Geografia e Estatística. Censo Demográfico 2010. [Acesso em 2014 jun 03]. Disponível em: URL: http://www.ibge.gov.br/home/estatistica/populacao/censo2010/>

11. Reis LA, Oliveira EM, Oliveira TA, Caires R, Santos BS. Perfil sociodemográfico e de saúde do idoso em Instituição de Longa Permanência para Idosos em Vitória da Conquista/BA. InterScientia 2013 set-dez;1(3):50-9

12. Colembergue JP, Conde SR. Uso da Miniavaliação Nutricional em idosos institucionalizados.Scientia Medica 2011; $21(2): 59-63$.

13. Gomes RC, Pinto CS, Soar C. Prevalência de desnutrição em idosos Institucionalizados. XIV Encontro Latino Americano de Iniciação Científica e X Encontro Latino Americano de Pós-Graduação; São Paulo: Universidade do Vale do Paraíba; 2011.

14. Silva AKQ, Gusmão SC, Castro KR, Moreira RAN, Morais AHA. Perfil nutricional de idosos assistidos em instituição de longa permanência na cidade de Natal, RN. Geriatria \& Gerontologia 2010;4(1):27-35.

15. Volpini MM, Frangella VS. Avaliação nutricional de idosos institucionalizados. Einstein 2013;11(1):32-40.

16. PazRC, Fazzio DMG, Santos ALB. Avaliação nutricional em idosos institucionalizados. Revisa 2012 Jan-Jun;1(1): 9-18.

17. Frank AA, Soares E.A. Nutrição no envelhecer. Atheneu, 2004.

18. Araújo MAR, Lima LS, Ornelas GC, Logrado MHG. Análise comparativa de diferentes métodos de triagem nutricional do paciente internado. Com. Ciências Saúde 2010;21(4):331-42. 